\title{
Application Status of Gynecological Endoscopy and Related Issues
}

\author{
Xiaojun Liu ${ }^{1}$, Lili Xuan*,1 \\ ${ }^{1}$ China-Japan Union hospital of Jilin University, Changchun, Jilin, 130033 \\ * corresponding author
}

Keywords: Gynecological Endoscopy; application status; related issues

\begin{abstract}
Gynecologic endoscopy is a revolution in surgery and is undergoing rapid development. It is not only the technical branch of surgery, but has gradually become the mainstream of modern surgery. It has become the three basic techniques of gynecological surgery together with open surgery and vaginal surgery, considered to be a sign of minimally invasive surgery. Endoscopic technology combines advanced science and technology with modern medicine to expand and extend the surgeon's vision and arms, and at the same time change our thinking concepts, technical routes and operating skills. It is becoming a necessary skill for gynecologists in the 21st century.
\end{abstract}

\section{Introduction}

In recent years, through the unremitting efforts and daring practice of many endoscopic researchers, gynecologic endoscopic techniques have become more mature and have made considerable progress. Some even predicted that "there is no laparoscopic surgery" [1]. Of course, this may not be excessive for a few "top masters," but for most endoscopy doctors, laparoscopic surgery is not yet complete due to the limitations of current technology and equipment and the specificity of certain diseases. Instead of open surgery.

\section{Principles of Implementation and Development of Endoscopic Technology}

For example, the range of surgery for cervical cancer, the choice of radiotherapy and chemotherapy, the staging of endometrial cancer, the staging of ovarian cancer, and the cytoreductive surgery of tumors all have clear requirements, regardless of the surgical approach or surgical approach. All these requirements should be met. That is to say, the same surgery is performed in different ways, or minimally invasive surgery can be performed to achieve minimally invasive effects. The choice of indications is actually four elements, namely the patient and his illness, the surgeon and his technique, not a simple disease that is suitable for a particular surgical procedure. Only four elements are fully fit, and it is a good choice. Otherwise, if any one is unsuitable, it should be changed or adjusted. Diseases and patients are problems to be considered in the diagnosis and treatment. The surgeons and surgical methods are also issues to be considered in the diagnosis and treatment. For endoscopic surgery, the concept, ability, and experience of the practitioner must not be forgotten. Any surgical technique and surgeon should not use surgery as a display of technology or equipment. Among them, the key is the surgeon, not the surgical method. A well-trained, skilled surgeon, a beautiful open surgery or vaginal surgery will also achieve ideal results. The choice of indications is relative, not absolute; it is limited, not unlimited. A doctor faces all kinds of patients and various technologies, one kind of technology, and one patient faces various doctors. The "matching" of them is clinical philosophy and art. As such individualized considerations, we must also pay attention to the wishes and requirements of patients and their families, and express humanistic care in the process of diagnosis and treatment. In talking with the sick family, we must show the advantages of minimally invasive endoscopic surgery, but also to explain its limitations, and possible problems, or the possibility of conversion to laparotomy. The doctor-patient communication choice should not be paternalistic but negotiated. This is even more important in the development of a newer technology. 


\section{Potential Medical Risks of Gynecologic Endoscopy}

It is well-known that CO2 pneumoperitoneum established during laparoscopic surgery can produce certain side effects on the human respiratory and circulatory system. The high solubility of CO2 and the pressure gradient of CO2 between the peritoneal cavity and the blood can lead to a rapid increase in $\mathrm{CO} 2$ absorption, causing hypercapnia and acidosis. Pneumoperitoneum can increase the intra-abdominal pressure. The pressure is $15 \mathrm{~mm} \mathrm{Hg}$ when the diaphragm lifts up and the lung function declines, especially when the patient is in the head and low position. The result is a physiological dead space increase and aeration-perfusion imbalance. In addition, increased intra-abdominal pressure can also lead to visceral vasoconstriction, decreased inferior vena cava, renal vein and hepatic venous return, thereby reducing the heart load. Increased intra-abdominal pressure can also increase the resistance of the entire peripheral blood vessels, increase the mean arterial pressure, and increase the cardiac afterload. When the intra-abdominal pressure is controlled at 8 to $12 \mathrm{~mm} \mathrm{Hg}$, the influence of the pneumoperitoneum on the circulatory system is at the boundary; when the intra-abdominal pressure increases to $16 \mathrm{mmHg}$, it can have a significant effect. At inflation pressures of up to $15 \mathrm{mmHg}$, cardiac output can be unchanged or slightly reduced due to compensatory increase in heart rate; once the increase is significant, cardiac output is significantly reduced. In addition, peritoneal dilatation may induce vasovagal reflexes, cardiac arrhythmias, and cardiac arrest. In addition to the above physiological effects, under the pressure of $15 \mathrm{~mm} \mathrm{Hg}, \mathrm{CO} 2$ pneumoperitoneum will cause changes in the ultrastructure of the peritoneum, which may promote the formation of metastasis at the puncture port of malignant tumor cells. We speculate that $\mathrm{CO} 2$ pneumoperitoneum is not conducive to laparoscopic surgery for malignant tumors. Studies have shown that the pneumoperitoneum formed by electronic pulsed pneumoperitoneum is widely used in clinical practice. This can lead to severe fluctuations in the intra-abdominal pressure during the operation. This may be caused by the instability of intra-abdominal pressure. The large impact, combined with the nature of CO2 pneumoperitoneum, determines that doctors and patients must face the potential risks associated with these unfavorable factors when performing laparoscopic surgery.

From the perspective of technical operation, although hysteroscopic surgery is easier to grasp and the complications are less than laparoscopic surgery, the high uterine pressure required for hysteroscopic surgery makes the complications of air embolism more prone to occur than laparoscopic surgery, and the mortality rate high. As early as 1997, there were statistics that 13 cases of definite diagnosis of air embolism occurred globally, and the mortality rate was as high as $69.23 \%$. In recent years, there have been reports of complications of air embolism in hysteroscopic surgery at home and abroad. Air embolism is mainly caused by outside air entering the blood circulation, especially the vaporization of tissues formed by electrosurgery, and through the open sinusoids into the veins, resulting in serious consequences of irreversibility. Air embolism has become the leading cause of death in patients undergoing hysteroscopic surgery in China.

\section{Preplans and Countermeasures of Gynecologic Endoscopy Technology to Prevent Risk}

As against all other medical risks, the potential risk prevention of gynecologic endoscopy should also be an effective and operational quality management model. In view of the various potential medical risks and causes of these gynecological endoscopic techniques, we believe that the core of risk prevention lies in improving the quality of gynecologic endoscopic surgery. This is an eternal theme. In order to improve the quality of gynecologic endoscopic surgery, we must look for defects from the sources of management systems, medical rules and regulations, and basic training for medical staff, and improve them, strengthen communication between doctors and patients, correctly select indications, and improve doctors' gynecological endoscopic surgery operation level and strengthen Equipment and device management is an important guarantee for the successful prevention of medical risks in gynecologic endoscopic surgery.

Good doctor-patient communication is very important for the patient's psychological, physical condition assessment and treatment prognosis. The basis for doctor-patient communication is the 
following: patient listening, solid professional knowledge, and harmonious doctor-patient relationship. Doctors should be cautious about all relevant patient information. To make doctor-patient communication effective and effective, it must be that the patient feels that she is fully informed about her problems and participates in discussions about her own treatment plan. Because gynecological diseases are private and highly individualized, it takes doctors special sensitivity to obtain patient honest answers during the interview process. The "high-tech nature" of endoscopic techniques also often makes patients "cloudy". If patients have doubts about the technique before surgery or lack of understanding, they often cause dissatisfaction with the medical process, increase anxiety and frustration. The difficulty of the treatment process will increase, the probability of treatment failure will increase, and the patient will respond to the lack of treatment. Therefore, before performing gynecologic endoscopic surgery, patients should fully and objectively explain the indications of the surgery, the general procedure of the surgery, and the contents of the informed informed consent of the procedure. Inform patients that endoscopic surgery is not a "mysterious technique" and that it can be controlled by a doctor so that the trust of patients and their families can be obtained. We emphasize communication between doctors and patients for specific surgical treatments. They should not be performed just before surgery, but should begin when the patient needs a gynecological endoscopic surgery for the first outpatient visit. The sign of achieving good communication between doctors and patients is that patients understand that the risks in the medical process are shared by doctors and patients. Despite careful preoperative preparation and preplanning, some complications still have a certain incidence. The more difficult the operation, the higher the incidence of complications. Once the complications occur, on the premise of ensuring the safety of the patient's life, as far as possible in the surgical process to make up for it, at the same time to explain the patient's family condition in detail, is strictly prohibited to conceal, to ensure that patients and their families informed consent right. If some complications can not be remedied in the same surgery, you need to perform a second operation, but also to the patient's family to explain the reasons for understanding.

Strengthen the training of gynecological endoscopy technology, lay a solid foundation, and continue to learn The selection of endoscopists must be subject to strict access systems, supervision systems, and standardized training systems. Pay attention to basic training, strictly follow the principle of endoscopic surgery grading training, to develop a qualified gynecological endoscopic doctor who can fully grasp all kinds of technical operations under the endoscope and have the ability to independently perform surgery. The preoperative assessment of the relationship between individual ability and the level of surgery required to perform the operation on a technical level requires that surgical indications be strictly selected on the premise of ensuring that there are no technical errors in the operation. Standardized training not only involves professional theory and technology, but also includes the cultivation of qualities such as knowledge of the humanities. Endoscopic surgery imposed on patients for certain personal purposes is strictly prohibited. Emphasis on the prospectiveness and predictability of endoscopic surgeons for surgical complications, and to have the initiative to guard against medical risks awareness and responsibility. It is necessary to establish a treatment plan for the occurrence of various accidents during surgery. For example, if the first puncture causes retroperitoneal great blood vessel damage, it must be converted immediately to open the abdomen to stop the bleeding quickly; once the patient undergoes drastic changes in vital signs during surgery, it should stop immediately. Surgical operation, stop the CO2 pneumoperitoneum or uterine distention, restore to non-pneuetic or non-inflated state, wait until the patient's vital signs are stable before considering whether to continue the endoscopic surgery; emergency equipment and instruments in the process of failure should be timely Replace spare equipment or take appropriate measures to ensure smooth operation.

Strictly manage endoscopic related surgical instruments and equipment, and ensure good working conditions of equipment and instruments A qualified endoscopic physician should be familiar with the performance of the used instruments and equipment, and act as endoscopic physician's "weapons”, good work for surgical instruments and equipment. The status is a guarantee of the successful completion of endoscopic surgery. Special management, cleaning and disinfection 
shall be conducted to register the use of various equipment, and the hidden troubles of equipment and equipment shall be checked and repaired in a timely manner. Even equipment such as power outlets should be included in regular inspection and maintenance projects. Put an end to the repeated use of disposable surgical instruments, otherwise it can easily cause cross-infection and cause serious consequences. For the laparoscopic surgical instruments that are easy to lose, our experience is to prepare two identical common surgical instruments in the same sterilizer, such as clamps, scissors, etc.; each device can also be individually sterilized by ethylene oxide. If a device is damaged during surgery, it can be replaced immediately without affecting the normal operation.

\section{Conclusion}

Surgical quality is an eternal topic in the discussion of gynecologic endoscopy. It is the key to preventing various risks of gynecologic endoscopic surgery. The improvement of the comprehensive quality of endoscopists, standardized medical behaviors, strong external guarantees, and awareness of full participation have a significant significance in reducing medical risks and improving medical quality. In order to further improve the quality of medical care and achieve the goals of safety, practicality, equality, and harmony in medical activities, gynecologic endoscopic physicians have a long way to go.

\section{References}

[1] Zakhari A, Czuzoj-Shulman N, Spence AR, et al, Laparoscopic and robot-assisted hysterectomy for uterine cancer: a compari-son of costs and complications[J]. Am J Obstet Gynecol, 2015, 213(5): $1-7$.

[2] Galaal K, Bryant A, Fisher AD, et al. Laparoscopy versus lapa-rotomy for the management of early stage endometrial cancer[J]. Cochrane Database Syst Rev, 2012, 9:CD006655.

[3] Juhasz-Böss I, Mallmann P, Möller CP, et al. Use of Laparosco-py in the treatment of endometrial and cervical cancer - resultsof a 2012 Germany-wide Survey[J]. Geburtshilfe Frauenheilkd, 2013, 73(9):911-917.

[4] Walker JL, Piedmonte MR, Spirtos NM, et al. Recurrence and survival after random assignment to laparoscopy versus laparoto-my for comprehensive surgical staging of uterine cancer: Gyne-cologic Oncology Group LAP2 Study[J]. J Clin Oncol, 2012, 30(7): 695-700.

[5] Gehrig PA, Cantrell LA, Shafer A, et al. What is the optimal min-imally invasiveurgical procedure for endometrial cancer stag-ing in the obese and morbidly obsese woman?[J]. Gynecol On-col, 2008, $111: 41-45$. 